\title{
Las facultades de medicina catalanas definen las competencias básicas comunes de los licenciados en medicina
}

\author{
Josep Carreras. Departament de Ciències Fisiológiques I. Facultat de Medicina. Universitat de Barcelona.
}

La Agencia para la Calidad del Sistema Universitario de Cataluña (AQU Catalunya) viene desarrollando diversas acciones a fin de dar soporte a las universidades catalanas en el proceso de construcción del espacio europeo de educación superior, entre las que destaca el denominado Programa DISSENY (Diseño). La construcción del espacio europeo representa un nuevo paradigma docente que comporta una profunda renovación de los planes de estudio de las diversas titulaciones, con definición de los perfiles de competencias profesionales y académicas, reformulación de los objetivos de formación, y uso de más variados y adecuados métodos de enseñanza/aprendizaje, y de evaluación de los estudiantes. En este contexto, el Programa DISSENY nació con dos grandes objetivos: desarrollar nuevos planes de estudios, y construir una metodología que permita orientar a los responsables académicos por lo que hace a los procedimientos a seguir para renovar los planes de estudios y para garantizar su calidad.

El principal instrumento metodológico del Programa es el desarrollo de una "Guía" que incluye el análisis de tres grandes elementos: a) el proceso de diseño del plan de estudios (evaluando los métodos empleados para el diseño del programa de formación y los procedimientos formales utilizados para su aprobación); b) las características del plan de estudios ( contemplando el perfil de formación, los objetivos generales del centro en relación con el plan, la estructuración en asignaturas, los métodos de evaluación y las condiciones de ingreso de nuevos alumnos); c) la capacidad y los recursos necesarios para el desarrollo del plan ( personal académico y PAS, servicios de soporte, instalaciones, sistemas de organización y coordinación de la enseñanza, sistemas de información).

Partiendo de esta Guía, y tomando como referentes los marcos legislativos, los marcos generales establecidos por AQU Catalunya, los estándares internacionales y los sistemas de garantía de la calidad, los catálogos de competencias básicas de los correspondientes titulados, y los sistemas de evaluación de dichas competencias, los responsables académicos diseñarán los nuevos planes de estudios. La evaluación ulterior de los planes deberá confirmar el cumplimiento de los requisitos necesarios con vistas a su certificación/acreditación.

Pues bien, hasta el presente tres titulaciones han firmado el correspondiente convenio con AQU Catalunya, y participan en el Programa DISSENY: Medicina, Psicología e Ingeniería Química. En el caso de la titulación de Medicina, se creó en el año 2003 un grupo de trabajo, constituido por un representante de cada una de las cuatro Facultades de medicina de Cataluña y dos representantes de AQU, que en una primera fase ha elaborado ya un documento sobre las competencias profesionales básicas que han de poseer todos sus licenciados. El documento se ha basado, fundamentalmente, en el referente internacional "Global Minimum Essential Requeriments" del "Institute for Internacional Medical Education". Ha adecuado la estructura y la terminología de este informe a la traducción al catalán y, teniendo en cuenta los catálogos de competencias ya existentes en la Facultades de Medicina de las Universidades de Barcelona y de Lérida así como la realidad social catalana, ha incorporado nuevos elementos que lo enriquecen. Distribuye las competencias en siete grandes ámbitos: "Valores profesionales, actitudes, comportamiento y ética", "Fundamentos científicos de la medicina", "Habilidades de comunicación", "Habilidades clínicas", "La salud de la población y los sistemas de salud", "El uso de la información", "Pensamiento crítico e investigación".

Después de ser sometido a estudio y debate por las Facultades de Medicina y por otras instituciones 
y agentes significativos en la formación de los graduados en Medicina, este documento ha de constituir la base para la definición de posibles competencias adicionales a nivel de cada Facultad, para la determinación de los correspondientes objetivos de formación y para el diseño de los sistemas de evaluación y de certificación de los estudiantes adecuados.

Cabe destacar que el grupo de trabajó asumió plenamente las competencias generales y transversa- les comunes a toda titulación universitaria, tal como los definió el Proyecto "TUNING”.

El documento completo y sus versiones en español e inglés, así como información oficial sobre el Programa DISSENY se hallan en la web de AQU Catalunya (www.aqucataluna.org). En la misma web pueden consultarse los números 19 y 21 de "El Butlletí. Butlletí d'informació sobre la qualitat universitària", que contienen información sobre el desarrollo del programa. 